\title{
La responsabilité sociale des facultés de médecine, un moyen de réconcilier les étudiants avec leur engagement médical
}

\author{
Social accountability of medical schools as a way of reconciling students \\ with their commitment to medicine
}

\begin{abstract}
Monsieur,
Le «Consensus mondial sur la responsabilité sociale des facultés de médecine " ${ }^{[1]}$ met en exergue la nécessité d'améliorer la capacité des facultés de médecine à répondre aux besoins et défis de la santé pour les citoyens et la société en général, en accord avec les valeurs de qualité, d'équité, de pertinence et d'efficience. Cette notion rappelle que la responsabilité médicale s'exerce au regard des besoins et des attentes de la société avant tout.

Par ailleurs, on connaît aujourd'hui l'évolution de la capacité des étudiants en médecine à faire preuve d'empathie au cours de leur cursus ${ }^{[2]}$. Alors que l'empathie s'efface, le cynisme gagne progressivement du terrain.
\end{abstract}

Ainsi, il semble plus que jamais nécessaire, d'un côté, de réaffirmer les finalités sociales et sociétales de la médecine et de la formation médicale initiale et, de l'autre, de recentrer l'action médicale et la formation des professionnels de santé sur la personne. Il s'agit là de deux constats distincts. À bien des égards, l'un et l'autre pourraient sembler un peu désabusés et il convient sûrement de s'interroger quant aux facteurs qui sont en cause. Une des hypothèses explicatives pourrait être en lien avec le décalage perçu par les étudiants entre leurs attentes et la réalité de la médecine qu'on leur propose en modèle.

Lors du récent congrès international d'éducation médicale, organisé à Lyon en août 2012 à l'initiative conjointe de la Société internationale francophone d'éducation médicale (SIFEM) et de l'Association for medical education in Europe (AMEE), un atelier proposé par des membres du comité de pilotage du projet francophone de recherche-action sur la responsabilité sociale des facultés de médecine a présenté cette initiative internationale. À notre grand étonnement, le public était pour moitié composé d'étudiants en médecine, qui avaient été interpellés par le titre de cet atelier. Un échange très intéressant a mis en évidence que, pour ceux-ci, la notion de responsabilité sociale était une évidence. Ils ont exprimé que, en fidélité à leur engagement initial en médecine, la notion de responsabilité sociale des facultés de médecine leur permettait de se réconcilier avec une pratique médicale centrée sur le patient et adaptée à ses attentes et ses besoins.

Il nous semble dès lors opportun de rappeler que cette recherche-action ambitieuse inclut à ce jour 28 facultés de médecine francophones localisées dans 14 pays. Son objectif général est d'expérimenter et d'évaluer la pertinence, l'applicabilité et la mise en œuvre d'une démarche de qualité, inspirée des principes de responsabilité sociale définis par le Consensus mondial ${ }^{[1,3]}$ et d'en démontrer l'utilité en tant que stratégie de choix pour améliorer l'impact des facultés de médecine et de la formation médicale sur la santé.

Trois phases sont prévues dans le cadre de ce projet :

La phase 1 d'applicabilité cherche à évaluer la pertinence et l'implication des facultés de médecine, telles qu'elles seront perçues par l'ensemble des 
partenaires de la faculté. Elle permettra d'identifier des normes et des indicateurs pour la phase 2 d'expérimentation. Celle-ci souhaite évaluer leur validité et leur opérabilité selon le modèle ConceptualisationProduction-Utilisabilité $(\mathrm{CPU})^{[4]}$. Enfin, la phase 3 d'évaluation permettra d'apprécier les effets et l'impact de la mise en œuvre du processus et du recours à ces indicateurs à plus ou moins long terme, le but final étant la mise en place d'un système d'accréditation $^{[4]}$ en responsabilité sociale des facultés.

Nous pensons que la démarche développée dans le cadre de cette recherche-action autour du principe de la responsabilité sociale des facultés de médecine pourrait rejoindre les préoccupations des jeunes médecins en formation, en leur fournissant un cadre conceptuel approprié à leur engagement médical, centré sur l'exercice du soin, au service des patients et de la société. Ils pourraient y trouver une justification des deux finalités au regard desquelles ils se sont engagés : soigner les maladies (to cure) et prendre soin (to care). "Or ces deux soins sont tous deux nécessaires. On exerce toujours un soin et sur quelqu'un. Or n'exercer que l'un des deux c'est mutiler (le soin) ou être mutilant (la personne)» ${ }^{[5]}$.

Comme le souhaitait Jean Bernard, cité par Hervé $^{[6]}$, « l'éthique de la biologie et de la médecine doit s'intégrer à une réflexion de grande ampleur sur le plan politique, et la servir». Parce qu'il met en exergue les valeurs de qualité, d'équité, de pertinence et d'efficience, le principe de responsabilité sociale des facultés de médecine réaffirme fondamentalement que la formation des professionnels de santé répond à une exigence éthique. À ce titre, l'engagement dans des réalisations pratiques développées en référence au concept de responsabilité sociale, tel que le projet de recherche-action déjà évoqué, est une opportunité fortement porteuse de sens pour des étudiants et nous voulons croire à leur engagement dans ce projet.

Le principe de responsabilité sociale anticipe une évolution souhaitable des systèmes de santé pour plus de pertinence et d'efficience, dans lesquels les futurs médecins et les professionnels de santé en général trouveraient toute leur place. C'est pourquoi, à titre personnel, nous ne pouvons qu'encourager nos enseignants à faire connaître ce projet et à y impliquer d'autres étudiants.

Il faut poursuivre l'effort d'information pour tenter de faire opposition à la fuite vers le cynisme au profit de l'empathie retrouvée, dans un processus de réconciliation avec la vie bonne de Paul Ricœur: «Une vie bonne avec et pour les autres, dans le respect des institutions justes. " ${ }^{[7]}$.

Benoit Valentin TUDREJ

Interne en médecine générale Faculté de médecine de Poitiers 40 rue Rabelais, Poitiers, France Mailto: benoit.tudrej@gmail.com

\section{Références}

1. Contribution collective de la conférence pour le consensus mondial sur la responsabilité sociale des facultés de médecine. Consensus mondial sur la responsabilité sociale des facultés de médecine. Pédagogie Médicale 2011;12:37-48.

2. Chen DCR, Kirshenbaum DS, Yan J, Kirshenbaum E, Aseltine RH. Characterizing changes in student empathy throughout medical school. Med Teach 2012;34:305-11.

3. Boelen C. Consensus mondial sur la responsabilité sociale des facultés de médecine. Santé Publique 2011;23:247-50.

4. Boelen C, Grand'Maison P, Ladner J, Pestiaux D. Responsabilité sociale et accréditation. Une nouvelle frontière pour l'institution de formation. Pédagogie Médicale 2008;9:235-44.

5. Worms F. Le moment du soin, à quoi tenons-nous ? Paris : Presses universitaires de France, 2010.

6. Hervé C. Ethique, politique et santé. Paris : Presses Universitaires de France, 2000.

7. Ricœur P. Soi-même comme un autre. Paris : Le Seuil, 1990. 